This item was submitted to Loughborough's Research Repository by the author.

Items in Figshare are protected by copyright, with all rights reserved, unless otherwise indicated.

\title{
Degradation mechanisms of bioresorbable polyesters. Part 1, Effects of random scission, end scission and autocatalysis
}

\section{PLEASE CITE THE PUBLISHED VERSION}

https://doi.org/10.1016/j.actbio.2013.12.039

\section{PUBLISHER}

Elsevier (@ Acta Materialia Inc.)

\section{VERSION}

AM (Accepted Manuscript)

\section{PUBLISHER STATEMENT}

This work is made available according to the conditions of the Creative Commons Attribution-NonCommercialNoDerivatives 4.0 International (CC BY-NC-ND 4.0) licence. Full details of this licence are available at: https://creativecommons.org/licenses/by-nc-nd/4.0/

\section{LICENCE}

CC BY-NC-ND 4.0

\section{REPOSITORY RECORD}

Gleadall, Andrew, Jingzhe Pan, Marc-Anton Kruft, and Minna Kellomaki. 2019. "Degradation Mechanisms of Bioresorbable Polyesters. Part 1, Effects of Random Scission, End Scission and Autocatalysis". figshare. https://hdl.handle.net/2134/26775. 


\section{Degradation mechanisms of bioresorbable polyesters. Part 1. Effects of random scission, end scission and autocatalysis}

Andrew Gleadall ${ }^{\mathrm{a}}$, Jingzhe Pan*a, and Marc-Anton Kruft ${ }^{\mathrm{b}}$

${ }^{a}$ Department of Engineering, University of Leicester, Leicester, LE1 7RH, UK

${ }^{\mathrm{b}}$ Purac Biomaterials, P.O. Box 21, 4200 AA Gorinchem, The Netherlands

* Corresponding author. Tel: +44 (0)116 223 1092; Fax: +44 (0)116 252 2525; E-mail: jp165@le.ac.uk;

The final publication is available at Elsevier via http://dx.doi.org/10.1016/j.actbio.2013.12.039

ABSTRACT: A mathematical model was developed to relate the degradation trend of bioresorbable polymers to different underlying hydrolysis mechanisms including noncatalytic random scission, autocatalytic random scission, noncatalytic end scission or autocatalytic end scission. The effect of each mechanism on molecular weight degradation and potential mass loss was analysed. A simple scheme was developed to identify the most likely hydrolysis mechanism based on experimental data. The scheme was firstly demonstrated using case studies and then used to evaluate data collected from 31 publications in the literature to identify the dominant hydrolysis mechanisms for typical biodegradable polymers. The analysis showed that most of the experimental data indicates autocatalytic hydrolysis as expected. However the study shows that the existing understanding on whether random or end scission controls degradation is inappropriate. It was revealed that pure end scission cannot explain the observed trend in molecular weight reduction because it would be too slow for end scission to reduce the average molecular weight. On the other hand pure random scission cannot explain the observed trend in mass loss because too few oligomers would be available to diffuse out of a device. It is concluded that the chain ends are more susceptible to cleavage which produces most of the oligomers leading to mass loss. However it is random scission that dominates the reduction in molecular weight.

Key words: Biodegradable polymers, biodegradation, random scission, end scission, modelling. 


\section{Introduction}

A large number of experiments have been conducted to understand the degradation of bioresorbable polymers such as polylactic acid (PLA), polyglycolic acid (PGA) and polycaprolactone (PCL) [1-31] which are used for various medical applications. A phenomenological mathematical model has been developed by Pan and his co-workers to predict the degradation rate of the biodegradable polymers [32-35]. It was demonstrated that the model is able to fit a wide range of experimental data for changes in molecular weight, mass and crystallinity as functions of degradation time. The purpose of this paper is to present a more detailed model that can be used to relate degradation behaviour to the underlying hydrolysis mechanisms. The mathematical model is used in order to understand the fundamental effects of each hydrolysis mechanism. It is not proposed to be able to predict degradation characteristics from initial material properties because the effects of many factors are not currently understood in enough detail.

The hydrolysis mechanisms being considered include random scission, end scission, noncatalytic hydrolysis and autocatalytic hydrolysis. In noncatalytic degradation, the ester bonds are cleaved in the presence of water whereas for autocatalytic degradation the hydrolysis reaction are catalysed by the carboxylic acid chain ends of water-soluble oligomers and monomers [1]. In random scission it is assumed that each ester bond in the polymer has an equal chance of chain cleavage whereas end scission assumes that only ester bonds at the end of polymer chains are cleaved. Experimental evidence for which hydrolysis mechanisms are dominant is conflicting due to the number of factors that affect degradation and inconsistency between experiments. Shih [36] suggested that end scission is dominant with approximately 10 times the rate of random scission. However for a high molecular weight sample, a single random scission has a greater impact on molecular weight than 1000 end scissions so their experiment actually indicates that random scission controls the molecular weight reduction. The experiment by Schliecker et al. [13] supports the theory of noncatalytic hydrolysis because it was found that the addition of oligomers does not accelerate degradation. Other experiments support the theory of autocatalytic hydrolysis [2, 37]. It has been widely observed that degradation occurs faster at the core of large samples compared to the surface because oligomers and monomers, which act as catalysts, diffuse out of the polymer near the surface [22]. Currently, there is no simple method of interpreting experimental data to identify the underlying hydrolysis mechanisms.

It has been suggested that a linear relationship between $\left(1 / M_{n}\right)$ and time indicates noncatalytic hydrolysis [38] and a linear relationship between $\left(1 / M_{n}\right)^{0.5}$ and time indicates autocatalytic 
hydrolysis [1, 37]. However, there is considerable experimental data in that literature that demonstrates a delay before the reduction of molecular weight [10, 22-29], and therefore does not fit either trend. The experiments of Antheunis et al. [27] demonstrate a delay trend when initial polymer chains do not possess carboxylic acid end groups but no delay when they do. The model here considers hydrolysis to only be catalysed by the acid chain ends of water soluble oligomers and monomers, not the chain ends of long chains which may be unable to catalyse hydrolysis due to lack of mobility or may initially not possess carboxylic acid end groups. One purpose of the current paper is to provide a wider interpretation of autocatalytic hydrolysis.

It is not fully understood which hydrolysis mechanisms are generally most prevalent in degradation experiments. In this paper, an analysis scheme is developed that can quickly identify which hydrolysis mechanisms are likely to be dominant based on experimental data for molecular weight and/or mass loss. The trends of molecular weight degradation and mass loss predicted by the mathematical model for various combinations of noncatalytic/autocatalytic hydrolysis and random/end scission are analysed and translated into the simple analysis scheme. Case studies demonstrate the use of the scheme and a large set of experimental data from the literature is evaluated to identify the dominant hydrolysis mechanisms. The particular focus of this study is predominantly on poly(lactide) and poly(glycolide) polymers in order to draw unambiguous conclusions regarding their degradation. However, the qualitative analysis scheme is not constrained to just poly(lactide) and poly(glycolide). The effects of initial molecular weight and residual monomer in relation to the hydrolysis mechanism are the subject of a separate paper [39].

\section{The mathematical model}

The phenomenological model developed by Pan and co-workers [32-34] is modified to separate the different hydrolysis mechanisms including noncatalytic random scission, autocatalytic random scission, noncatalytic end scission and autocatalytic end scission. The polymer is assumed to consist of amorphous polymer chains, oligomers, monomers and a crystalline phase. It is assumed that the crystalline phase, characterised by the degree of crystallinity $X_{c}$ (no units) strongly resists hydrolysis such that only the amorphous polymer chains suffer from hydrolysis chain scission. The rate of chain scission is determined by the concentrations of the reactants and catalyst. For random scission, the reactant is the ester bonds in amorphous chains which are characterised by the concentration $C_{e}\left(\mathrm{~mol} \mathrm{~m}^{-3}\right)$. For end scission the reactant is the amorphous chain ends characterised by $C_{\text {end }}\left(\mathrm{mol} \mathrm{m}^{-3}\right)$. It is assumed that water is always 
abundant [40] and its concentration does not affect the hydrolysis rate. The hydrolysis reaction can be catalysed by $\mathrm{H}^{+}$disassociated from the carboxylic acid end groups. Using $C_{\text {acid }}\left(\mathrm{mol} \mathrm{m}^{-}\right.$ ${ }^{3}$ ) to represent the concentration of the carboxylic end groups, the concentration of $\mathrm{H}^{+}$can be calculated as $C_{H^{+}}=\left(K_{a} C_{a c i d}\right)^{n}$ [34] where $K_{a}$ is the acid disassociation constant and $n$ (no units) is taken to be 0.5 as suggested by Siparsky et al. [37] indicating equilibrium condition for acid disassociation. We use $\left.R_{r s}(\mathrm{~mol} \mathrm{~m})^{-3}\right)$ and $R_{e s}\left(\mathrm{~mol} \mathrm{~m}^{-3}\right)$ to represent the molar concentrations for random and end scissions respectively. Following Han et al. [34] the rate of random scission is given by

$$
\frac{d R_{r s}}{d t}=k_{r 1} C_{e}+k_{r 2} C_{e}\left(\frac{C_{a c i d}}{1-X_{c}}\right)^{n}
$$

and the rate of end scission is given by

$$
\frac{d R_{e s}}{d t}=k_{e 1} C_{e n d}+k_{e 2} C_{e n d}\left(\frac{C_{a c i d}}{1-X_{c}}\right)^{n} .
$$

Here $k_{r 1}$ and $k_{e 1}\left(\right.$ day $\left.^{-1}\right)$ are the noncatalytic reaction constants and $k_{r 2}$ and $k_{e 2}\left(\left[\mathrm{~mol}^{-1} \mathrm{~m}^{3}\right]^{0.5} \mathrm{day}^{-}\right.$ ${ }^{1}$ ) are the autocatalytic reaction constants where subscripts $r$ and $e$ indicate random and end scission respectively. The acid disassociation constant $K_{a}$ has been merged into $k_{r 2}$ and $k_{e 2}$. The single rate equation for chain scission proposed by Han et al. [34] has been split into two equations so that the random and end scissions can be evaluated separately. The total scission concentration $R_{S}\left(\mathrm{~mol} \mathrm{~m}^{-3}\right)$ is then given by

$$
R_{s}=R_{r s}+R_{e s} \text {. }
$$

In end scission a monomer is produced by each scission and the production of monomers per unit volume $R_{m}\left(\mathrm{~mol} \mathrm{~m}^{-3}\right)$ is simply given by

$R_{m}=R_{e s}$.

In random scission an oligomer may be produced by chance if an ester bond near a chain end is cleaved. Following the statistical analysis by Flory [41] the production of ester units of oligomers per unit volume, $R_{o l}\left(\mathrm{~mol} \mathrm{~m}^{-3}\right)$, can be related to the concentration of random scissions $R_{r s}$ through

$$
\frac{R_{o l}}{C_{e 0}}=\alpha\left(\frac{R_{r s}}{C_{e 0}}\right)^{\beta}
$$

in which $C_{e 0}\left(\mathrm{~mol} \mathrm{~m}^{-3}\right)$ is the concentration of ester bonds in all phases at time $t=0$. The values $\alpha=28$ (no units) and $\beta=2$ (no units) apply if the oligomers are defined as short chains of less than 8 units [38] as assumed in this work. 
Assuming Fick's law of diffusion, the oligomer and monomer concentrations, $C_{o l}$ and $C_{m}$ (mol $\mathrm{m}^{-3}$ ), are governed by

$$
\frac{d C_{a}}{d t}=\frac{d R_{a}}{d t}+\operatorname{div}_{x_{i}}\left(\underset{x_{i}}{\left.\operatorname{grad}\left(C_{a}\right)\right)}\right.
$$

in which the nomenclature of vector analysis is used and the subscript $a$ is either $m$ to represent monomer diffusion or $o l$ to represent oligomer diffusion. The terms $R_{o l}$ and $R_{m}$ represent the concentrations of oligomers and monomers that have been produced due to chain scission whereas $C_{o l}$ and $C_{m}\left(\mathrm{~mol} \mathrm{~m}^{-3}\right)$ represent the current concentrations due to both production and diffusion. A finite difference scheme is implemented for the spatial discretisation of the second term on the right hand side in Eq. (6). The diffusion coefficient $D\left(m^{2} d a y^{-1}\right)$ depends on the porosity and crystallinity of the polymer. It is calculated based on the diffusion coefficient of the polymer $D_{0}\left(m^{2} d a y^{-1}\right)$ and of pores $D_{\text {pore }}\left(m^{2} d a y^{-1}\right)$ as discussed in the work of Han and Pan [33].

Pan and co-workers [32-34] further assumed that only the oligomers and monomers can catalyse the hydrolysis reaction because the carboxylic end groups of the long chains could be initially capped and the chains are not mobile. $C_{\text {acid }}$ in Eqs. (1) and (2) can then be calculated as

$$
C_{\text {acid }}=C_{m}+\left(C_{o l} / m\right)
$$

in which $m$ (no units) is the average degree of polymerisation of the oligomers. In this study we have set $m=4$ because oligomers are assumed to have less than 8 units of degree of polymerisation. The molar concentration of polymer chains $N_{\text {chain }}\left(\mathrm{mol} \mathrm{m}^{-3}\right)$ is given by

$$
N_{\text {chain }}=N_{\text {chain } s 0}+\left(R_{r s}-\left(R_{\text {ol }} / m\right)\right)
$$

in which $N_{\text {chainso }}\left(\mathrm{mol} \mathrm{m}^{-3}\right)$ is the initial molar concentration of chains and $C_{\text {end }}=2 N_{\text {chain }}$ in Eq. (2).

According to Avrami [42-44], the degree of crystallinity $X_{c}$ can be calculated through the extended degree of crystallinity $X_{\text {ext }}$ (no units) by

$$
\frac{d X_{c}}{d X_{e x t}}=X_{\max }-X_{c}
$$

in which $X_{\max }$ (no units) has been introduced to set a limit for the maximum degree of crystallinity. Gleadall et al. [35] showed that the extended degree of crystallinity can be related to the concentration of chain scission $R_{S}$ using

$$
X_{e x t}=p_{x} \eta_{A} R_{s} V_{c}
$$


in which $p_{x}$ (no units) is the probability of crystallisation of a cleaved chain, $\eta_{A}$ is Avogadro's constant $\left(\mathrm{mol}^{-1}\right), V_{c}\left(\mathrm{~m}^{3}\right)$ is the volume of a single polymer crystallite. During biodegradation amorphous polymer chains are consumed by oligomer production, monomer production and crystallisation which leads to

$$
C_{e}=C_{e 0}-\left(R_{o l}+R_{m}+\omega X_{c}\right)
$$

where $\omega$ (no units) is the inverse molar volume of crystalline phase.

The number-averaged molecular weight, $M_{n}\left(\mathrm{~g} \mathrm{~mol}^{-1}\right)$, can be calculated as

$$
M_{n}=\frac{\left(C_{e}+\omega X_{c}\right) M_{0}}{N_{\text {chain }}}
$$

in which $M_{0}\left(\mathrm{~g} \mathrm{~mol}^{-1}\right)$ is the molar mass of each polymer repeat unit. In the molecular weight calculation, oligomers and monomers are excluded because they are too small to be detected by typical measuring techniques such as gel permeation chromatography. Each random scission increases the chain number by one but has a probability to produce $R_{o l} / \mathrm{m}$ number of oligomers. For end scission, $N_{\text {chain }}$ remains constant and $M_{n}$ reduces due to the reduction of amorphous ester units $C_{e}$. In contrast, for random scission, the main factor for $M_{n}$ reduction is the increase in the number of chains.

Eqs. $(1-12)$ are numerically integrated using the direct Euler scheme giving the molecular weight, degree of crystallinity, and concentrations of oligomers and monomers as functions of degradation time. Although there are a large number of parameters in the model, several are directly related to each other, and many are not adjusted between simulations. The values of $\alpha$ and $\beta$ are calculated according to Flory's most probably distribution in all simulations, as derived in previous work [34], and therefore only depend on the value of $m$. The terms $p_{x}$ and $V_{c}$ for crystallisation combine into a single parameter and are only kept separate to preserve their physical meanings. The terms $C_{e} 0$ and $\omega$ are always equal and the chosen value represents an assumption that the densities of poly lactic acid amorphous and crystalline phases are both $1250 \mathrm{~kg} / \mathrm{m}^{3}$.

The parameters that have the greatest effect on the degradation are $k_{r 1}, k_{r 2}, k_{e 1}, k_{e 2}$, and $n$. They affect the rate of chain scission which in turn affects molecular weight, crystallinity, and mass loss. Increasing the reaction rates increases the rate of chain scission for each hydrolysis mechanism. Increasing $n$ has the effect of accelerating autocatalytic hydrolysis. The values of $X_{\max }, p_{x}$, and $V_{c}$ control crystallinity and do not significantly affect other aspects of degradation. The values of $m, D_{0}$ and $D_{\text {pore }}$ affect the size and diffusion of small chains and therefore affect mass loss and the rate of autocatalytic hydrolysis. The parameters that are typically adjusted in 
order to fit experimental data for molecular weight are $k_{r 1}, k_{r 2}, k_{e l}$, and $k_{e 2}$. If crystallinity data is also being fitted, the parameters $X_{\max }$ and $p_{x}$ are also adjusted to fit the data. And when fitting mass loss data, the parameters $D_{0}$ and $D_{\text {pore }}$ are adjusted for the best fitting too.

\section{Degradation trends by different hydrolysis mechanisms}

The change in molecular weight and accumulation of oligomers and monomers as functions of time are computed using the mathematical model for different combinations of random or end scission with autocatalytic or noncatalytic hydrolysis. For simplicity we focused on amorphous polymer in this section and use a typical set of data for PLA of $M_{0}=72 \mathrm{~g} \mathrm{~mol}^{-1}, N_{\text {chaino }}=4.35$ mol m${ }^{-3}$ and $C_{e}=17300 \mathrm{~mol} \mathrm{~m}^{-3}$ which represents an initial molecular weight of $M_{n}=286,000$ $\mathrm{g} \mathrm{mol}^{-1}$. When presenting the data, the molecular weight is normalised by its initial value and the degradation time is normalised by a characteristic time $t_{c}$ which is the time taken for the molecular weight to reduce to $10 \%$ of its initial value. Consequently the absolute values of the reaction constants do not have an effect on the presented results and only their relative values are important. Also, if the rate of $M_{n}$ reduction accelerates with time, the curve will appear to have a slower initial rate of $M_{n}$ reduction but such an interpretation is invalid because the curve is constrained to pass through the point of $10 \% M_{n}$ at normalised time $=1$. For molecular weight - time curves, only the shapes of curves should be compared. The oligomers and monomers are water soluble and may diffuse out of a device. The diffusion of these water soluble chains is not considered in this section because its effect on degradation was fully studied by Wang et al. [32]. The accumulation of oligomers and monomers together is referred to as small chains and used as an indication for potential mass loss. In the current paper the initial concentrations of oligomers and monomers are set to be an extremely small value of $10^{-10} C_{e 0}$, which is just $10^{-8}$ weight percentage. Such a small value is used in order to generate curves that clearly highlight the trends from autocatalytic to noncatalytic in this section. The effect of this initial value on the degradation behaviour is the topic of the accompanying paper [39] so is not discussed here. However, the reader should be aware that some experiments may have residual monomer contents as high as several per cent, which would result in the curves shifting by some degree from autocatalytic to noncatalytic in appearance. The experimental data fittings presented in the next section can still be achieved if a high initial monomer content is used. 


\subsection{Random scission and the effect of autocatalytic strength}

Fig. 1 shows the transition from noncatalytic to autocatalytic hydrolysis assuming only random scission occurs (by setting $k_{e l}=k_{e 2}=0$ in the model). The ratio of $k_{r 2} / k_{r l}$, which reflects the relative strength of autocatalysis to non-autocatalysis, is set at 0, 5, 20, 100, 500 and infinity. It can be observed from the figure that a strong autocatalytic hydrolysis is characterised by a delay in the reduction of the molecular weight while a weak autocatalytic hydrolysis is characterised by an initially sharp reduction in the molecular weight. This is because for a strong autocatalytic hydrolysis, the rate of chain scission accelerates greatly as small chains build up while noncatalytic chain scission does not accelerate during degradation. The pure noncatalytic random scission curve (A) is the only curve which gives a linear line on a plot of $\left(1 / M_{n}\right)$ versus time.

Another important observation from the figure is that the accumulation of oligomers and monomers as a percentage of total ester units is insignificant until the molecular weight reaches a very small value. They do however affect the degradation rate. The number of carboxylic end groups of these small and water soluble chains are sufficient enough to significantly alter the behaviour in the molecular weight reduction. However any measurable mass loss would not be expected before the polymer breaks apart if its degradation occurs entirely by random scission. This is because random scission is very inefficient to produce oligomers but quite efficient to reduce the molecular weight. Autocatalytic hydrolysis is associated with relatively early oligomer production (measurable at normalised time $\approx 1.5$ ) while very few oligomers are produced in noncatalytic hydrolysis (negligible even at normalised time $=10$ ). This simply reflects the fact that molecular weight reduces at a greater rate in the latter stages of degradation for autocatalytic hydrolysis. For random scission, the small chain fraction depends mainly on the absolute value of molecular weight. For a purely random scission model setup $\left(k_{e l}=k_{e 2}=0\right)$, oligomers account for $1 \%$ of the total polymer when $M_{n}$ has reduced to approximately $5000 \mathrm{~g}$ $\mathrm{mol}^{-1}$. A simple Monte Carlo random chain scission simulation also gives similar results. 


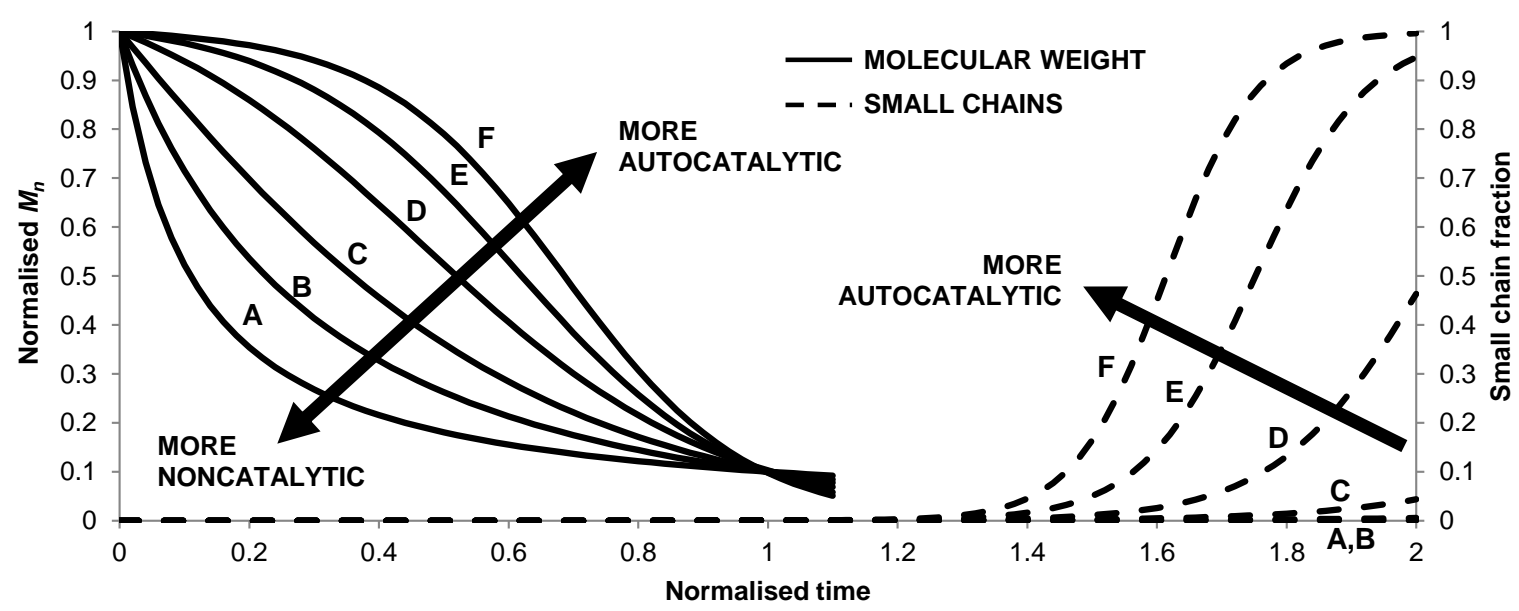

Fig. 1. Random scission simulations with variable degrees of autocatalysis. Normalised $M_{n}$ (solid lines) and the sum weight fraction of oligomers plus monomers (dashed lines) are shown versus normalised time. The autocatalytic:noncatalytic rate ratios $\left(k_{r_{2}} / k_{r 1}\right)$ are $0(\mathrm{~A}), 5(\mathrm{~B}), 20$ (C), $100(\mathrm{D}), 500(\mathrm{E})$, and infinity (F).

\subsection{End scission and the effect of autocatalytic strength}

Fig. 2 shows the transition from noncatalytic to autocatalytic degradation assuming only end scission occurs $\left(k_{r 1}=k_{r 2}=0\right)$. The ratio of $k_{e 2} / k_{e l}$ is set as $0,0.02$ and infinity. Similar to random scission, strong autocatalytic hydrolysis is characterised by an initial delay in the molecular weight reduction. However no sharp initial reduction in molecular weight can be observed in any of the cases. This is because each end scissions has the same effect on molecular weight throughout degradation whereas the effect of each random scission reduces as the number of chains increases, which is discussed in detail in part 2 [39].

In strong contrast to random scission, the accumulation of oligomers and monomers occurs very early. This means significant mass loss could be expected if the degradation is controlled by end scission. It can also be observed that stronger autocatalytic hydrolysis delays the production of small chains. Both the reduction in molecular weight and production of short chains are delayed in strong autocatalytic hydrolysis due to lack of the catalyst (short chains) at the start of degradation. Noncatalytic hydrolysis does not require the short chains as a catalyst so does not demonstrate a delay. 


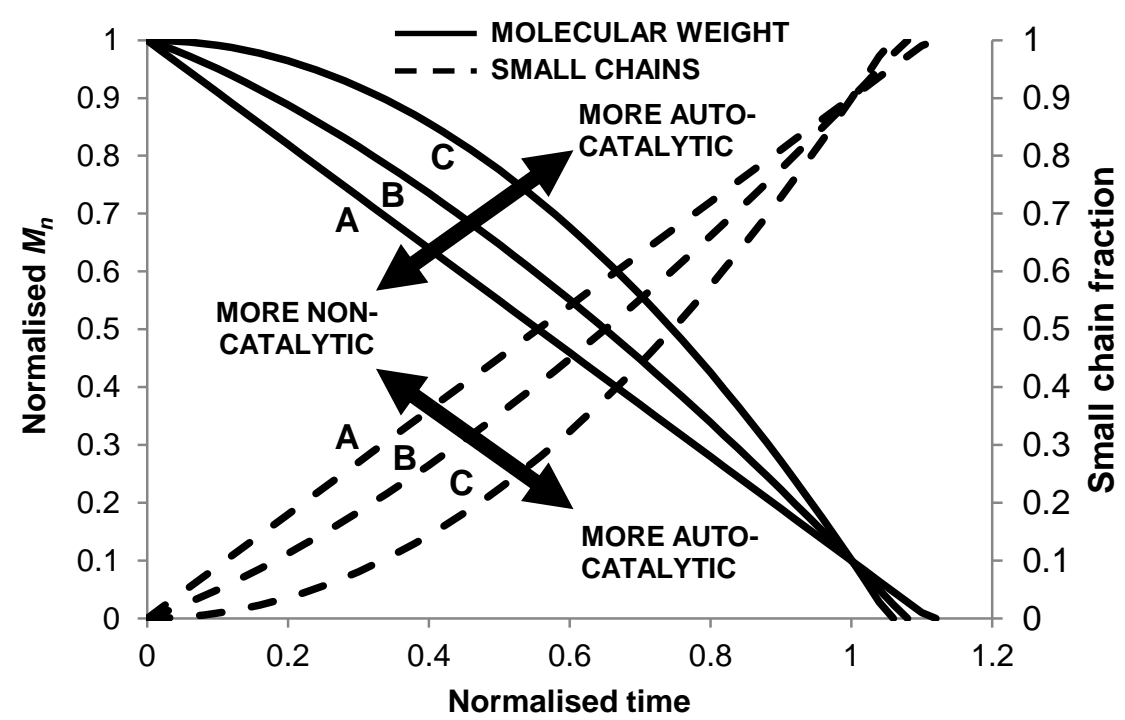

Fig. 2. End scission simulations with variable degrees of autocatalysis. Normalised $M_{n}$ (solid lines) and the sum weight fraction of oligomers plus monomers (dashed lines) are shown versus normalised time. The autocatalytic:noncatalytic rate ratios $\left(k_{e 2} / k_{e l}\right)$ are $0(\mathrm{~A}), 0.02(\mathrm{~B})$, and infinity (C).

\subsection{Autocatalytic hydrolysis and the transition from random to end scission}

Fig. 3 shows the transition from random to end scission assuming autocatalytic hydrolysis $\left(k_{r l}=k_{e l}=0\right)$. The ratio of $k_{e 2} / k_{r 2}$, which reflects relative strength of end scission to random scission, is set as $10^{2}, 10^{3}, 10^{4}, 5 \times 10^{5}$ and infinity. The case for pure random scission is already shown in Fig. 1 and omitted here for clarity.

It can be observed from Fig. 3 that random scission is characterised by a deceleration (the concave section on the curve) in the reduction rate of molecular weight at some stage of the degradation, which can also be clearly observed in Fig. 1. No such deceleration can be observed for the end scission cases (Figs. 2 and 3). It is also interesting to focus on cases $A, B$ and $C$ in Fig. 3. A difference of two orders of magnitude in the ratio of $k_{e 2} / k_{r 2}$ has very little effect on the molecular weight behaviour but greatly affects the production of oligomers and monomers and hence potential mass loss. This once again highlights that the effect of the underlying hydrolysis mechanisms may be significant on one aspect of the degradation behaviour but undetectable on a different aspect. The fraction of acidic chain ends present in oligomers as opposed to monomers is negligible even for low levels of end scission. An interesting observation is therefore that the rate of autocatalytic random scission is most likely determined 
by monomers so although molecular weight reduction is due to random scissions, the rate of reduction is controlled by end scission.

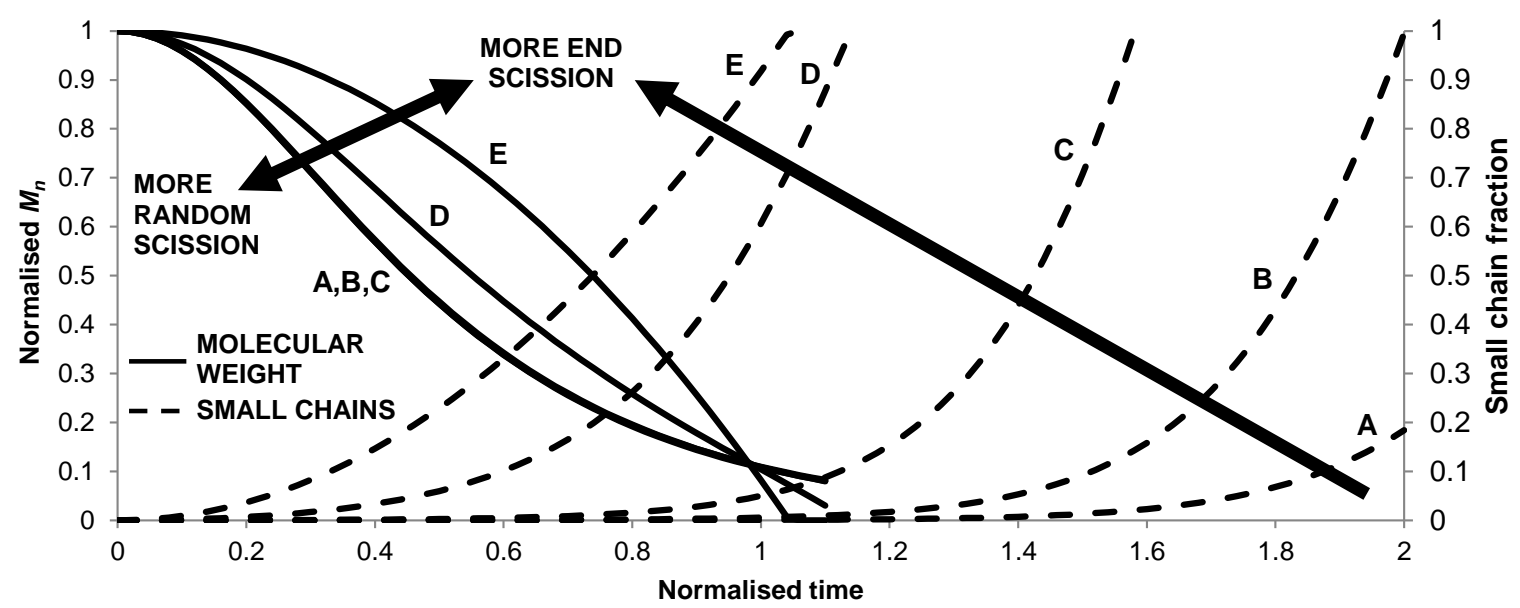

Fig. 3. Autocatalytic hydrolysis simulations with variable ratios of end scission to random scission reaction rates. Normalised $M_{n}$ (solid lines) and the sum weight fraction of oligomers plus monomers (dashed lines) are shown versus normalised time. The end:random scission rate ratios $\left(k_{e 2} / k_{r 2}\right)$ are $10^{2}(\mathrm{~A}), 10^{3}(\mathrm{~B}), 10^{4}(\mathrm{C}), 5 \times 10^{5}(\mathrm{D})$, and infinity $(\mathrm{E})$.

\subsection{Noncatalytic hydrolysis and the transition from random to end scission}

Fig. 4 shows the transition from random to end scission assuming noncatalytic hydrolysis $\left(k_{r 2}=k_{e 2}=0\right)$. The ratio of $k_{e 1} / k_{r 1}$, which reflects the relative strength of end scission to random scission, is set as $0,1 \times 10^{4}, 5 \times 10^{4}, 1 \times 10^{5}, 5 \times 10^{5}$ and infinity. A threshold for the end scission rate can also be observed from cases A and B for the molecular weight behaviour but not for the accumulation of the small chains. Examining the small chain accumulation as a function of time and comparing Figs. 3 and 4, it can be observed that autocatalytic hydrolysis is characterised by a delay in the accumulation of the small chains hence a delay in mass loss while noncatalytic hydrolysis is characterised by almost linear increase in the amount of oligomers and monomers.

\section{A qualitative scheme to identify dominant hydrolysis mechanisms and case studies}

When analysing experimental data, a set of values for parameters in the mathematical model can be found that provide the best fit between the model prediction and the data. This set of parameters can then be used to identify the underlying hydrolysis mechanism, for example, a 


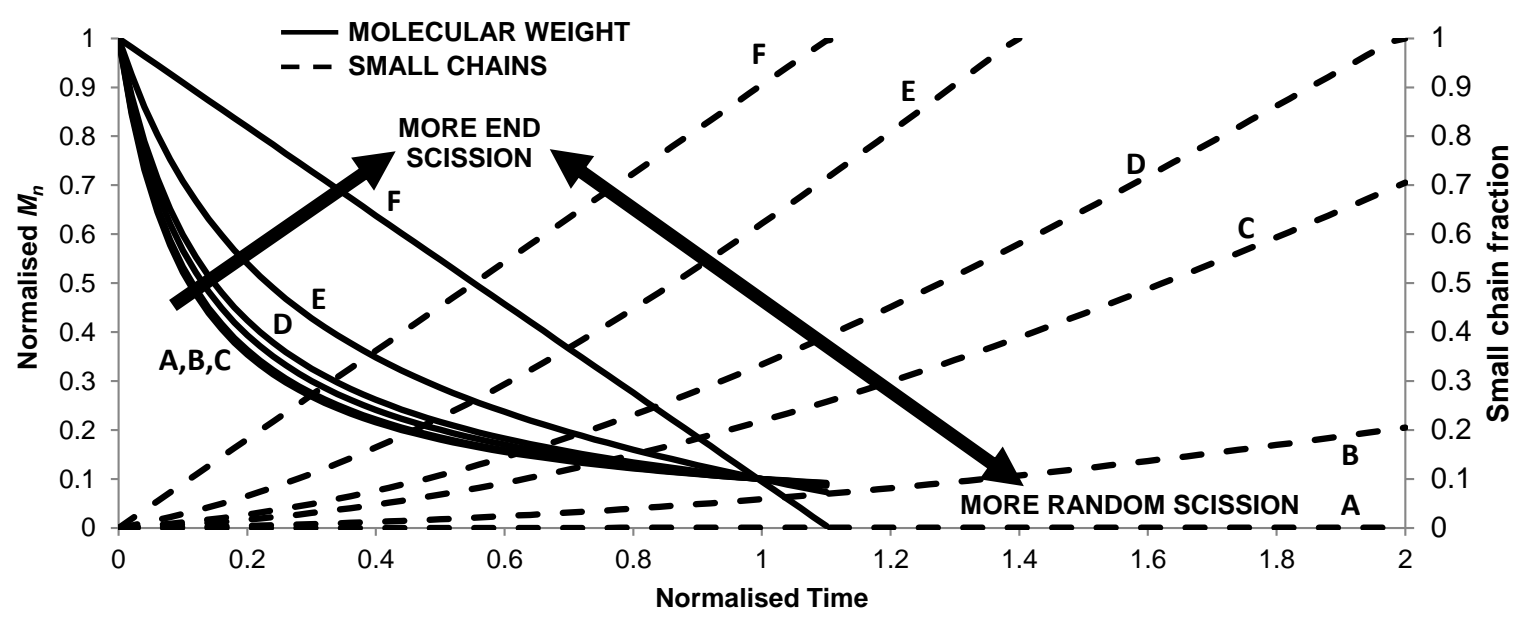

Fig. 4. Noncatalytic hydrolysis simulations with variable ratios of end scission to random scission reaction rates. Normalised $M_{n}$ (solid lines) and sum weight fraction of oligomers plus monomers (dashed lines) are shown versus normalised time. The end:random scission rate ratios $\left(k_{e l} / k_{r 1}\right)$ are $0(\mathrm{~A}), 1 \times 10^{4}(\mathrm{~B}), 5 \times 10^{4}(\mathrm{C}), 1 \times 10^{5}(\mathrm{D}), 5 \times 10^{5}(\mathrm{E})$, and infinity $(\mathrm{F})$.

large ratio of $k_{e 2} / k_{r 2}$ would indicate end scission dominates. However the analysis presented in section 3 provides a quick and qualitative analysis to identify the dominant hydrolysis mechanism. In this section both approaches are used to analyse two sets of experimental data obtained from the literature. This serves two purposes: (a) validation of the mathematical model and (b) a demonstration of the analysis scheme.

The analysis in section 3 can be briefly summarised as

i. A deceleration (concave section) on the molecular weight - time curve indicates random scission. Lack of the deceleration indicates end scission.

ii. A linear relationship between $\left(1 / M_{n}\right)$ and time indicates noncatalytic random scission without autocatalytic random scission. A nonlinear relationship indicates autocatalytic hydrolysis. An initial delay in the reduction of the molecular weight indicates a greater contribution from autocatalytic hydrolysis.

iii. Significant mass loss while $M_{n}>5000 \mathrm{~g} \mathrm{~mol}^{-1}$ indicates end scission.

iv. A linear increase in mass loss with time indicates noncatalytic end scission.

Because mass loss also requires the small chains to diffuse out of the specimen, a lack of mass loss does not necessarily indicate that end scissions do not occur. Similarly, a delay in mass loss does not prove autocatalysis. Points (i)-(iv) can be used as a simple and quick scheme to 
identify the underlying hydrolysis mechanisms. Fig. 5 shows schematically the typical regimes of the molecular weight behaviour. Only curve A would be linear on a plot of $\left(1 / M_{n}\right)$ versus time.

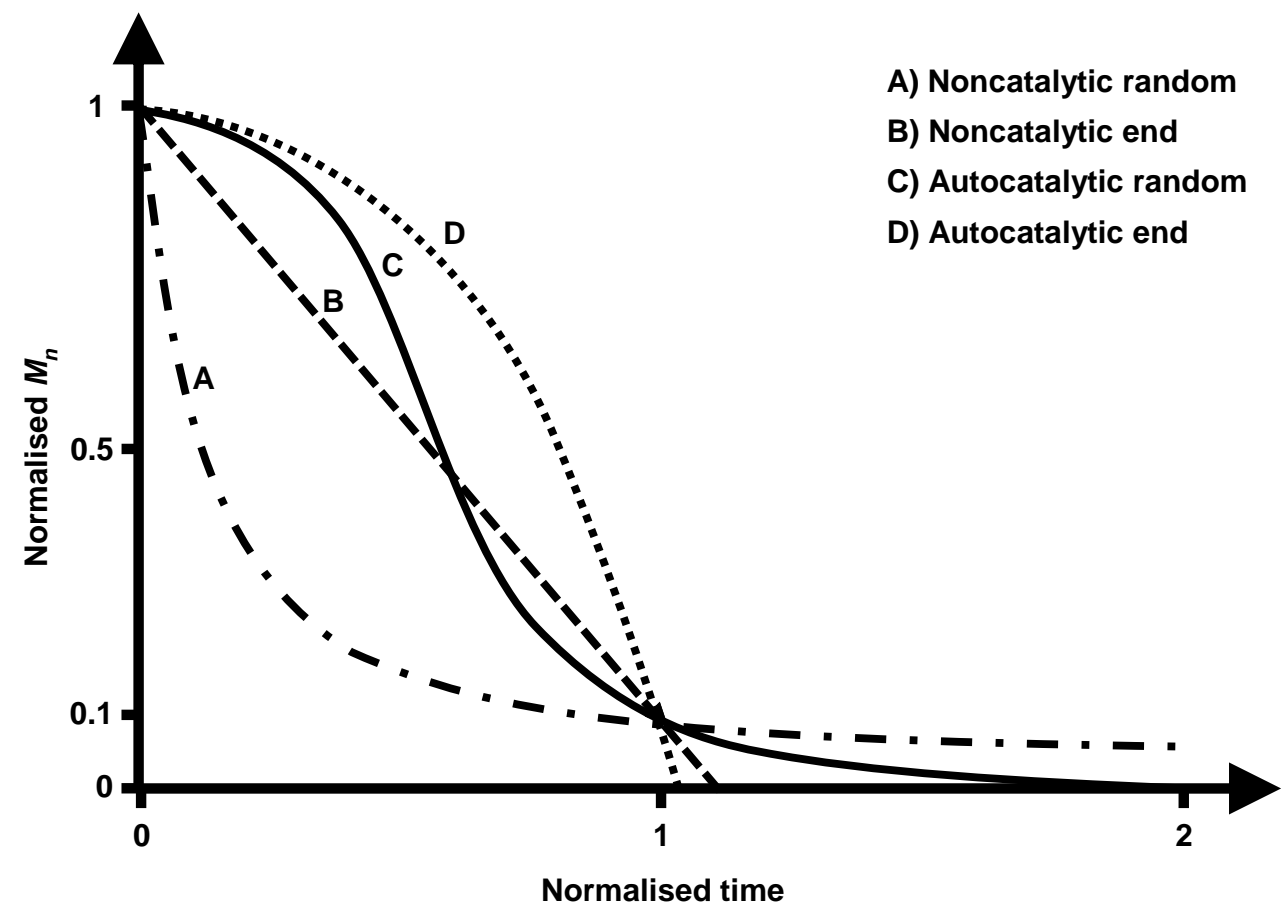

Fig. 5. Typical curves for normalised $M_{n}$ versus normalised time $\bar{t}$ for various theories of hydrolysis: (A) noncatalytic random scission, (B) noncatalytic end scission, (C) autocatalytic random scission, and (D) autocatalytic end scission.

\subsection{Case study A}

Beslikas et al. [25] carried out a set of degradation experiments using thin films of semicrystalline poly(lactide) in phosphate buffer solution (PBS) $\mathrm{pH} 7.2$ at $50^{\circ} \mathrm{C}$. Their experimental results are re-produced using discrete symbols in Fig. 6 showing normalised number averaged molecular weight, degree of crystallinity and mass loss as functions of time.

Significant mass loss while $M_{n}>5000 \mathrm{~g} \mathrm{~mol}^{-1}$ indicates that end scission must have happened. However a gentle deceleration can be observed on the molecular weight - time curve which indicates random scission also occurred. A plot of $\left(1 / M_{n}\right)$ versus time is not linear and an initial delay on the molecular weight - time curve can be observed which indicate that strong autocatalytic hydrolysis was in operation. It can therefore be concluded that the degradation is by a combination of autocatalytic random and autocatalytic end scission. 
The best fitting between the mathematical model and the data is shown in Fig. 6 using solid lines. The fitting parameters are provided in Table 1. It is not possible to achieve an equally good fitting with a different set of hydrolysis mechanisms. Both crystallisation and diffusion of small chains are included in the numerical model. Crystallite size $V_{c}$ is estimated from the literature [45]. It can be observed that the mathematical model can fit the data very well for the average molecular weight and degree of crystallinity and quite well for mass loss. The best fit is achieved by $k_{r l}=k_{e l}=0$, which indicates that the hydrolysis is fully autocatalytic, and supports the qualitative analysis. The reaction rate ratio of $k_{e 2 /} k_{r 2}=7500$ indicates that end scission occurs much faster than random scission. The high rate of end scission is necessary to produce the level of mass loss observed in the experiment. However it is very important to point out that the relatively small but finite random scission rate has a major effect on the molecular weight reduction. The dashed line in Fig. 6 shows the model prediction using identical set of parameters except that $k_{r 2}$ was set as zero. It can be observed that the small amount of random scission has a large effect on both molecular weight reduction and mass loss. A fitting to mass loss may be achieved without random scission by increasing $k_{e 2}$, but a fitting of molecular weight is not possible. Similarly, a fitting to mass loss is only possible if end scission is included. Both random and end scission are required to fit the data.

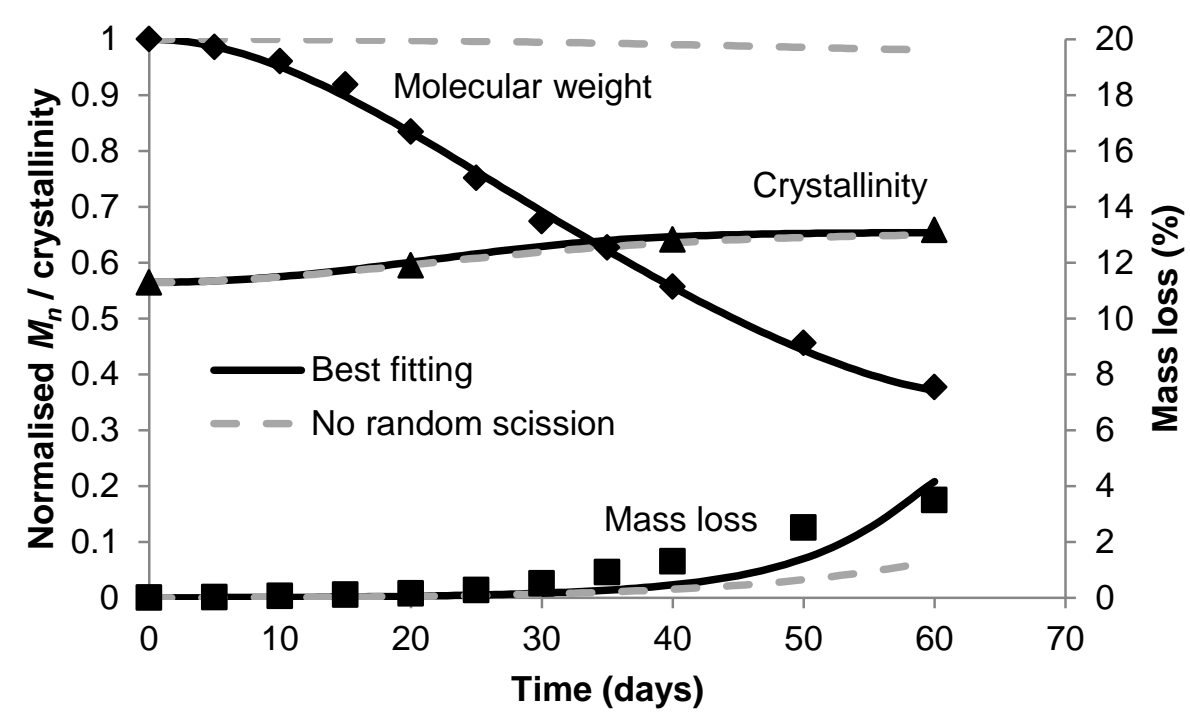

Fig. 6. A model fitting of molecular weight, crystallinity, and mass loss for combined autocatalytic random scission and autocatalytic end scission as suggested by the qualitative analysis (solid black lines). For comparison, a fitting without random scission also included (dashed grey lines). Discrete symbols indicate experimental data [25]. 


\subsection{Case study B}

Batycky et al. [26] carried out degradation experiments of drug-encapsulating microspheres made of poly(DL-lactide-co-glycolide) 50:50. The samples are amorphous throughout. Their data are reproduced in Fig. 7 showing normalised number averaged molecular weight and mass loss as functions of time. From the figure it can be observed that there is clear deceleration on the molecular weight - time curve indicating random scission. There is an initial delay in the molecular weight reduction and therefore a plot of $\left(1 / M_{n}\right)$ versus time is not linear which indicates autocatalytic random scission. However, the delay is less significant compared to case A, perhaps suggesting a noncatalytic hydrolysis contribution. The mass loss is significant while $M_{n}>5000 \mathrm{~g} \mathrm{~mol}^{-1}$ indicating a large end scission rate. The degradation is therefore through a combination of autocatalytic random and noncatalytic end scission. Batycky et al. [26] also used a mathematical model to determine whether end or random scission was dominant and concluded that a combination of both mechanisms was required to fit their data.

The solid lines in Fig. 7 show the best fitting of the model. All the parameters used in the fitting are provided in Table 1. Again it can be observed that the model is able to fit the experimental data very well. The best fit was obtained by setting $k_{r l}=k_{e 2}=0$ which indicates noncatalytic hydrolysis and $k_{e l} / k_{r 2}=1.26 \times 10^{5}$ which indicates end scission occurs much faster than random scission. Similar to Case A, the large end scission rate is necessary for the observed mass loss and a similar fitting cannot be achieved by using a different combination of hydrolysis mechanisms. However the small but finite rate of random scission is critical to the molecular weight reduction. The dashed lines in Fig. 7 show the model prediction using an identical set of parameters except that $k_{r l}$ was set to zero. As with Case A, it can be observed that the small amount of random scission effects both molecular weight reduction and mass loss. Random scission is again required to achieve a fitting to the experimental data for molecular weight and end scission is required for mass loss. The values of diffusion coefficients $D_{0}$ and $D_{\text {pore }}$ are chosen to give the best model fitting. They give an indication of the polymer diffusion coefficients. Significant variation is to be expected between different setups since many factors affect diffusion. For example, the experiments of Yoon et al. [46] found diffusion coefficients to be 3 orders of magnitude greater for water molecules in poly(lactide) than poly(glycolide). 


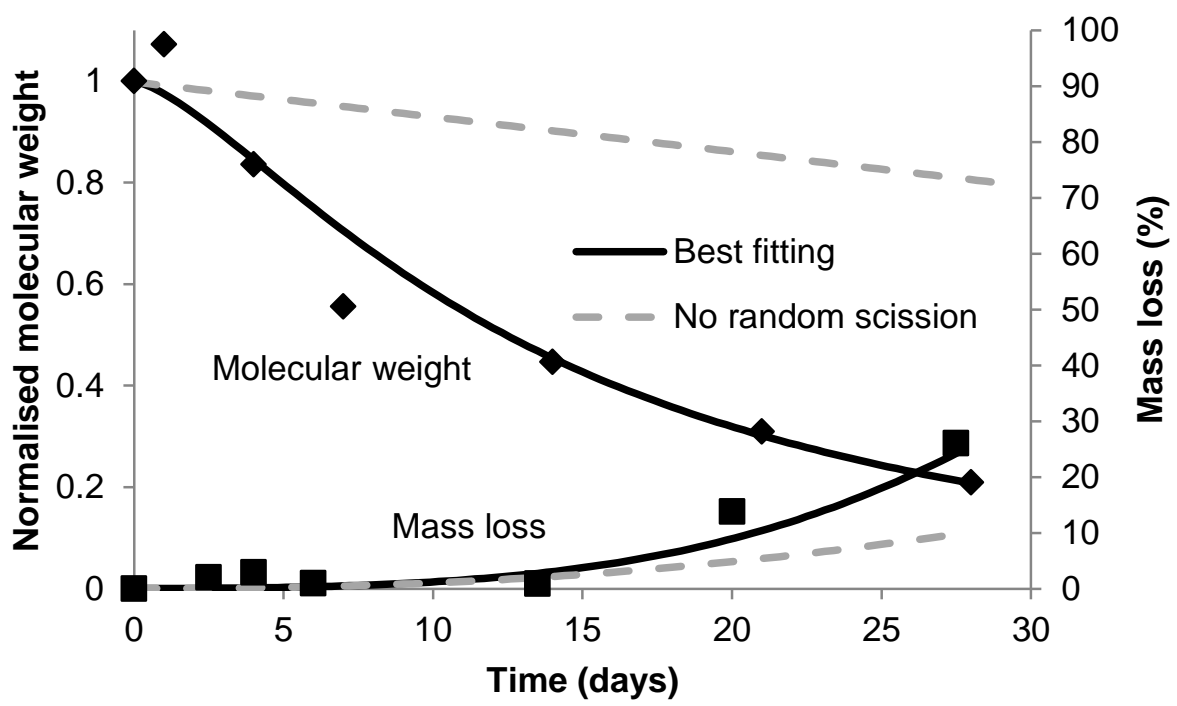

Fig. 7. A model fitting using a combination of autocatalytic random scission and noncatalytic end scission (solid black lines) as suggested by the qualitative analysis. A fitting without random scission is included for reference (dashed grey lines). Experimental data [26] for molecular weight and mass loss are discrete symbols.

Table 1. Values of the model parameters used in the fittings

\begin{tabular}{|c|c|c|c|}
\hline Model parameters & units & Case study A & Case study $B$ \\
\hline$M_{0}$ & $\mathrm{~g} \mathrm{~mol}^{-1}$ & 72 & $65^{\text {(a) }}$ \\
\hline$k_{r 1}$ & day $^{-1}$ & 0 & 0 \\
\hline$k_{r 2}$ & {$\left[\mathrm{~mol}^{-1} \mathrm{~m}^{3}\right]^{0.5} \mathrm{day}^{-1}$} & $3.0 \times 10^{-6}$ & $8.5 \times 10^{-6}$ \\
\hline$k_{e 1}$ & day $^{-1}$ & 0 & $1.26 \times 10^{5 *} k_{r 1}$ \\
\hline$k_{e 2}$ & {$\left[\mathrm{~mol}^{-1} \mathrm{~m}^{3}\right]^{0.5} \mathrm{day}^{-1}$} & $7500 * k_{r 2}$ & 0 \\
\hline$N_{\text {chainso }}$ & $\mathrm{mol} \mathrm{m}^{-3}$ & 10.4 & 55 \\
\hline$D_{0}$ & $\mathrm{~m}^{2}$ day $^{-1}$ & $2.5 \times 10^{-11}$ & $1.6 \times 10^{-15}$ \\
\hline$D_{\text {pore }}$ & $\mathrm{m}^{2}$ day $^{-1}$ & $2.5 \times 10^{-7}$ & $1.6 \times 10^{-11}$ \\
\hline Initial porosity & no units & 0 & 0 \\
\hline$C_{e 0}$ & $\mathrm{~mol}^{-1} \mathrm{~m}^{3}$ & 17,300 & 17,300 \\
\hline$\omega$ & $\mathrm{mol}^{-1} \mathrm{~m}^{3}$ & 17,300 & 17,300 \\
\hline$X_{\max }$ & no units & 0.655 & 0 \\
\hline$p_{x}$ & no units & 0.004 & 0 \\
\hline$V_{c}$ & $\mathrm{~m}^{3}$ & $4.19 \times 10^{-24}$ & 0 \\
\hline Initial $M_{n}$ & $\mathrm{~g} \mathrm{~mol}^{-1}$ & 120000 & 20500 \\
\hline Initial $X_{c}$ & no units & 0.5645 & 0 \\
\hline Film thickness or microsphere radius & $\mu \mathrm{m}$ & $50^{(\mathrm{b})}$ & 10 \\
\hline
\end{tabular}

(a) Molar mass is taken as the average of poly(lactide) and poly(glycolide)

(b) Film thickness is not given in the publication so estimated at $50 \mu \mathrm{m}$. 


\section{Review of hydrolysis mechanisms for experimental data available in the literature}

The qualitative analysis was applied to experimental data in 31 publications that we could obtain from the literature [1-31]. Detailed fitting with the mathematical model was also performed for typical cases. Table 2 lists the identified hydrolysis mechanisms for each individual paper. Experimental details such as polymer type, initial molecular weight and minimum dimension of the samples are also provided. The following conclusions can be drawn from the analysis:

- A combination of random and end scission is identified for almost all the data.

- Significant molecular weight reduction is always due to random scission.

- Mass loss is due to end scission except for thick samples (1.4-2.6mm) for which random scission may contribute.

- Autocatalytic random scission is required for molecular weight reduction in almost all publications.

Table 2 identifies the hydrolysis mechanisms that are required in order for a fitting to be achieved. Naturally, the best fitting will be achieved by allowing the model the flexibility to include all hydrolysis mechanisms to a greater or lesser extent. An important finding is that a good fitting cannot be achieved without autocatalytic random scission in most cases. Since the analysis found a mixture of autocatalytic and noncatalytic hydrolysis, it is likely that both types of hydrolysis mechanism occur depending of the setup of a particular experiment. There have been several experimental publications that suggest autocatalytic hydrolysis plays a significant role in degradation under specific conditions. In particular, heterogeneous degradation of large samples has been attributed to autocatalysis [14, 15, 22, 27, 47, 48], as has the accelerated degradation of samples with high residual monomer [18, 23], along with the accelerated degradation of polymers with carboxylic acid end groups versus benzyl alcohol end groups [27]. However, the findings of the analysis in Table 2 suggest that autocatalytic hydrolysis has a more important role than noncatalytic hydrolysis over a very broad range of conditions. The experiments considered in the analysis vary greatly in factors such as sample size and shape, initial molecular weight, polymer or copolymer type, buffer solution type and temperature, crystallinity, and the rate of degradation. But the model almost always suggests that autocatalytic hydrolysis occurs. It is important to note that since end scission is expected, the monomers that result from end scission control the rate of autocatalytic random scission, and therefore the rate of $M_{n}$ reduction. This is due to the fact that the number of monomers is significantly greater than oligomers, as discussed in Section 3. Since end scission has little or 
no effect on molecular weight, models that derive the acid catalyst concentration from the value of molecular weight do not consider the expected situation that end scission controls the concentration of acid catalyst. Also, an alternative interpretation of the findings of Antheunis et al. [27] that carboxylic acid end groups accelerate degradation versus benzyl alcohol end groups could be that the benzyl alcohol end groups are more resistant to end scission so the initial production of monomers, and therefore catalyst, is retarded.

In the most practical polymers, the initial molecular weight is too large for random scission to produce enough oligomers by chance in order to give the observed mass loss. However random scission is crucial in order to give the observed molecular weight reduction. Considering the experimental data for mass loss presented in Figs. 6 and 7 [25, 26], for the molecular weight to reduce by $67 \%$ only two random scissions per chain are required. It is impossible for this number of random scissions to produce $3 \%$ and $15 \%$ mass of oligomers by chance to give the observed mass loss given that the initial chains contain 1667 and 315 polymer units respectively. If there is no end scission, the observed mass loss would require that the polymer chains become water soluble at $M_{n} \approx 14000$ and $M_{n} \approx 2600 \mathrm{~g} \mathrm{~mol}^{-1}$ respectively. These values are much larger than those typically found in the literature which may be in the region of $<1000 \mathrm{~g}$ $\mathrm{mol}^{-1}[27,49,50]$. Random scission is only predicted to contribute significantly to mass loss in publications that used thick samples. This may suggest that random scission is more susceptible to autocatalysis than end scission because there is likely to be a higher concentration of oligomers and monomers in the centre of large samples than at the surface or than in smaller samples where they can more easily diffuse out of the polymer.

It can be generally concluded that ester bonds towards the end of polymer chains are more susceptible to hydrolysis than those in the middle. If end scission is due to the acidic chain end folding back on itself, it may be the case that a number of bonds near the chain end can be cleaved. Experimental measurements of lactic acid monomers would not identify oligomers produced by this type of end scission so may falsely be interpreted as evidence for random scission. 
Table 2. The model is used on a number of degradation experiments to determine the dominant hydrolysis mechanisms

\begin{tabular}{|c|c|c|c|c|c|c|c|}
\hline \multirow[b]{3}{*}{ Ref } & \multirow[b]{3}{*}{ Polymer type } & \multirow[b]{3}{*}{$\begin{array}{l}\text { Initial } M_{n} \\
\left(\mathrm{~kg} \mathrm{~mol}^{-1}\right)\end{array}$} & \multirow[b]{3}{*}{$\begin{array}{l}\text { Minimum } \\
\text { size }(\mathbf{m m})\end{array}$} & \multicolumn{4}{|c|}{ Scission type } \\
\hline & & & & \multicolumn{2}{|c|}{ Random scission } & \multicolumn{2}{|c|}{ End scission } \\
\hline & & & & $\begin{array}{c}\text { Auto- } \\
\text { catalytic }\end{array}$ & $\begin{array}{c}\text { Non- } \\
\text { catalytic }\end{array}$ & $\begin{array}{c}\text { Auto- } \\
\text { catalytic }\end{array}$ & $\begin{array}{c}\text { Non- } \\
\text { catalytic }\end{array}$ \\
\hline [1] & PLA 70L:30L,D & 290 & 1 & YES & & \multicolumn{2}{|c|}{ ONE OF THESE TWO } \\
\hline [2] & PLA - L, D, or L/D & 500 & 0.1 & YES & MAYBE & MAYBE & MAYBE \\
\hline [3] & PLGA 50DL:50G & 10.5 & N/A & YES & & & YES \\
\hline [4] & PLGA & 33 & 0.05 & YES & \multicolumn{3}{|c|}{ YES } \\
\hline [5] & PLLA & 550 & 0.05 & YES & & \multicolumn{2}{|c|}{ ONE OF THESE TWO } \\
\hline [6] & PLLA & 155 & 0.8 & YES & \multicolumn{3}{|c|}{ YES } \\
\hline [7] & PLLA & 166 & 0.8 & YES & & \multicolumn{2}{|c|}{ ONE OF THESE TWO } \\
\hline & PLLA & 100,150 & $1.5-3$ & YES & & \multicolumn{2}{|c|}{ ONE OF THESE TWO } \\
\hline [8] & PDLLA & $27-177$ & $1.5-3$ & YES & MAYBE & MAYBE & MAYBE \\
\hline [9] & PLA - L, D, or L/D & 90 & 0.05 & YES & \multicolumn{3}{|c|}{ YES } \\
\hline [10] & PLA - L, D, or L/D & 450 & 0.05 & YES & \multicolumn{3}{|c|}{ YES } \\
\hline$[11]$ & PLLA & 45 & 0.033 & & YES & & YES \\
\hline [12] & PLA 50L:50D & 450 & 0.05 & YES & & \multicolumn{2}{|c|}{ ONE OF THESE TWO } \\
\hline [13] & PLGA - 50DL:50G & 14 & 0.2 & YES & & & YES \\
\hline$[14]$ & PDLLA & 85 & 1.5 & YES & \multicolumn{3}{|c|}{ YES } \\
\hline [15] & PLLA & 72 & 2 & & YES & & YES \\
\hline [16] & PLGA & 53 & 0.2 & YES & & \multicolumn{2}{|c|}{ ONE OF THESE TWO } \\
\hline$[17]$ & PLLA & 550 & 0.05 & YES & & \multicolumn{2}{|c|}{ YES } \\
\hline [18] & PLGA 85:15 & $\mathrm{IV}=1.4 \mathrm{dl} / \mathrm{g}$ & $1.6-3.4$ & YES & & \multicolumn{2}{|c|}{ ONE OF THESE TWO } \\
\hline [19] & PLA 96L:4D & 37 & 2.6 & YES & & \multicolumn{2}{|c|}{ ONE OF THESE TWO } \\
\hline [20] & PLA 70L:30DL & $\approx 20$ & 2 & YES & & \multicolumn{2}{|c|}{ ONE OF THESE TWO } \\
\hline [21] & 90PLA:10PCL & 28 & 0.4 & YES & & & YES \\
\hline [22] & PDLLA & $20-34$ & 2 & YES & & \multicolumn{2}{|c|}{ ONE OF THESE TWO } \\
\hline [23] & PDLLA & 10 & 0.5 & YES & & \multicolumn{2}{|c|}{ YES } \\
\hline [24] & PLGA 50:50 & 28 & $0.0005-0.022$ & & YES & \multicolumn{2}{|c|}{ ONE OF THESE TWO } \\
\hline [25] & PLLA & 120 & thin film & YES & & \multicolumn{2}{|c|}{ YES } \\
\hline [26] & PLGA 50:50 & 20 & microsphere & YES & & & YES \\
\hline$[27,28]$ & PLA, PLGA, PCL & 10 & $1.4-2.3$ & YES & & & \\
\hline [29] & PLGA & 40 & 0.05 & YES & & & \\
\hline [30] & PDLA & 100 & 0.1 & YES & & \multicolumn{2}{|c|}{ ONE OF THESE TWO } \\
\hline [31] & PLLA & 160 & 2 & MAYBE & YES & MAYBE & MAYBE \\
\hline
\end{tabular}

\section{Conclusions}

The revised mathematical model was developed to consider degradation by the individual hydrolysis mechanisms noncatalytic random scission, autocatalytic random scission, noncatalytic end scission and autocatalytic end scission. The model was able to fit all experimental degradation data at hand. Simple qualitative trends in the degradation of molecular weight and mass loss were found to relate to the underlying hydrolysis mechanisms. These trends are that: 1) a deceleration of molecular weight reduction versus time indicates random scission whereas a lack of the deceleration indicates end scission; 2) noncatalytic hydrolysis is indicated by a linear relationship between $\left(1 / M_{n}\right)$ and time whereas a nonlinear relationship indicates autocatalytic hydrolysis; 3) mass loss while the polymer is still medium to high molecular weight indicates end scission; and 4) a linear increase in mass loss with time indicates noncatalytic end scission. 
The experimental degradation data from 31 publications was analysed to identify the most likely hydrolysis mechanisms using either the qualitative analysis mentioned above or detailed model fittings. The analysis found that: 1) a combination of random and end scission is almost always predicted to occur; 2) molecular weight reduction is always due to random scission; 3) mass loss is due to end scission except for thick samples for which random scission may contribute; and 4) autocatalytic hydrolysis is expected more often than noncatalytic hydrolysis. The effects of initial molecular weight and residual monomer are important but are not investigated in this paper to maintain simplicity. They are analysed in detail in part 2 [39] of this series of publications.

\section{Acknowledgements}

Andrew Gleadall acknowledges an EPSRC PhD studentship.

\section{References}

[1] Lyu, Schley J, Loy B, Lind D, Hobot C, Sparer R, et al. Kinetics and Time-Temperature Equivalence of Polymer Degradation. Biomacromolecules 2007;8:2301-10.

[2] Tsuji H. Autocatalytic hydrolysis of amorphous-made polylactides: effects of L-lactide content, tacticity, and enantiomeric polymer blending. Polymer 2002;43:1789-96.

[3] Schliecker G, Schmidt C, Fuchs S, Kissel T. Characterization and in vitro degradation of poly(2,3-(1,4-diethyl tartrate)-co-2,3-isopropyliden tartrate). Journal of Controlled Release 2004;98:11-23.

[4] Tan HY, Widjaja E, Boey F, Loo SCJ. Spectroscopy techniques for analyzing the hydrolysis of PLGA and PLLA. Journal of Biomedical Materials Research Part B: Applied Biomaterials 2009;91B:433-40.

[5] Tsuji H, Mizuno A, Ikada Y. Properties and morphology of poly(L-lactide). III. Effects of initial crystallinity on long-term in vitro hydrolysis of high molecular weight poly(L-lactide) film in phosphate-buffered solution. Journal of Applied Polymer Science 2000;77:1452-64.

[6] Weir N, Buchanan F, Orr J, Dickson G. Degradation of poly-L-lactide. Part 1: in vitro and in vivo physiological temperature degradation. Proceedings of the Institution of Mechanical Engineers, Part H: Journal of Engineering in Medicine 2004;218:307-19.

[7] Weir N, Buchanan F, Orr J, Farrar D, Dickson G. Degradation of poly-L-lactide. Part 2: increased temperature accelerated degradation. Proceedings of the Institution of Mechanical Engineers, Part H: Journal of Engineering in Medicine 2004;218:321-30. 
[8] Migliaresi C, Fambri L, Cohn D. A study on the in vitro degradation of poly(lactic acid). Journal of Biomaterials Science, Polymer Edition 1994;5:591-606.

[9] Tsuji H. In vitro hydrolysis of blends from enantiomeric poly(lactide)s. Part 1. Well-stereocomplexed blend and non-blended films. Polymer 2000;41:3621-30.

[10] Tsuji H. In vitro hydrolysis of blends from enantiomeric poly(lactide)s. Part 4: well-homocrystallized blend and nonblended films. Biomaterials 2003;24:537-47.

[11] Lam KH, Nieuwenhuis P, Molenaar I, Esselbrugge H, Feijen J, Dijkstra PJ, et al. Biodegradation of porous versus non-porous poly(L-lactic acid) films. Journal of Materials Science: Materials in Medicine 1994;5:181-9.

[12] Tsuji H, Del Carpio CA. In Vitro Hydrolysis of Blends from Enantiomeric Poly(lactide)s.

3. Homocrystallized and Amorphous Blend Films. Biomacromolecules 2002;4:7-11.

[13] Schliecker G, Schmidt C, Fuchs S, Wombacher R, Kissel T. Hydrolytic degradation of poly(lactide-co-glycolide) films: effect of oligomers on degradation rate and crystallinity. International Journal of Pharmaceutics 2003;266:39-49.

[14] Li S, McCarthy S. Further investigations on the hydrolytic degradation of poly (DLlactide). Biomaterials 1999;20:35-44.

[15] Li SM, Garreau H, Vert M. Structure-property relationships in the case of the degradation of massive poly( $\alpha$-hydroxy acids) in aqueous media. Journal of Materials Science: Materials in Medicine 1990;1:198-206.

[16] Cai Q, Shi G, Bei J, Wang S. Enzymatic degradation behavior and mechanism of Poly(lactide-co-glycolide) foams by trypsin. Biomaterials 2003;24:629-38.

[17] Tsuji H, Ikada Y. Properties and morphology of poly(L-lactide) 4. Effects of structural parameters on long-term hydrolysis of poly(L-lactide) in phosphate-buffered solution. Polymer Degradation and Stability 2000;67:179-89.

[18] Paakinaho K, Heino H, Väisänen J, Törmälä P, Kellomäki M. Effects of lactide monomer on the hydrolytic degradation of poly(lactide-co-glycolide) $85 \mathrm{~L} / 15 \mathrm{G}$. Journal of the Mechanical Behavior of Biomedical Materials 2011;4:1283-90.

[19] Niemelä T. Effect of $\beta$-tricalcium phosphate addition on the in vitro degradation of selfreinforced poly-1,d-lactide. Polymer Degradation and Stability 2005;89:492-500.

[20] Niiranen H, Pyhältö T, Rokkanen P, Kellomäki M, Törmälä P. In vitro and in vivo behavior of self-reinforced bioabsorbable polymer and self-reinforced bioabsorbable polymer/bioactive glass composites. Journal of Biomedical Materials Research Part A 2004;69A:699-708. 
[21] Vieira AC, Vieira JC, Ferra JM, Magalhães FD, Guedes RM, Marques AT. Mechanical study of PLA-PCL fibers during in vitro degradation. Journal of the Mechanical Behavior of Biomedical Materials 2011;4:451-60.

[22] Grizzi I, Garreau H, Li S, Vert M. Hydrolytic degradation of devices based on poly(dllactic acid) size-dependence. Biomaterials 1995;16:305-11.

[23] Hyon SH, Jamshidi K, Ikada Y. Effects of residual monomer on the degradation of DLlactide polymer. Polymer International 1998;46:196-202.

[24] Dunne M, Corrigan OI, Ramtoola Z. Influence of particle size and dissolution conditions on the degradation properties of polylactide-co-glycolide particles. Biomaterials 2000;21:1659-68.

[25] Beslikas T, Gigis I, Goulios V, Christoforides J, Papageorgiou GZ, Bikiaris DN. Crystallization Study and Comparative in Vitro-in Vivo Hydrolysis of PLA Reinforcement Ligament. International journal of molecular sciences 2011;12:6597-618.

[26] Batycky RP, Hanes J, Langer R, Edwards DA. A theoretical model of erosion and macromolecular drug release from biodegrading microspheres. Journal of Pharmaceutical Sciences 1997;86:1464-77.

[27] Antheunis H, van der Meer J-C, de Geus M, Kingma W, Koning CE. Improved Mathematical Model for the Hydrolytic Degradation of Aliphatic Polyesters. Macromolecules 2009;42:2462-71.

[28] Antheunis H, van der Meer J-C, de Geus M, Heise A, Koning CE. Autocatalytic Equation Describing the Change in Molecular Weight during Hydrolytic Degradation of Aliphatic Polyesters. Biomacromolecules 2010;11:1118-24.

[29] Raman C, Berkland C, Kim K, Pack DW. Modeling small-molecule release from PLG microspheres: effects of polymer degradation and nonuniform drug distribution. Journal of Controlled Release 2005;103:149-58.

[30] Pitt GG, Gratzl MM, Kimmel GL, Surles J, Sohindler A. Aliphatic polyesters II. The degradation of poly (DL-lactide), poly ( $\varepsilon$-caprolactone), and their copolymers in vivo. Biomaterials 1981;2:215-20.

[31] Pistner H, Bendi DR, Mühling J, Reuther JF. Poly (l-lactide): a long-term degradation study in vivo: Part III. Analytical characterization. Biomaterials 1993;14:291-8.

[32] Wang Y, Pan J, Han X, Sinka C, Ding L. A phenomenological model for the degradation of biodegradable polymers. Biomaterials 2008;29:3393-401.

[33] Han X, Pan J. A model for simultaneous crystallisation and biodegradation of biodegradable polymers. Biomaterials 2009;30:423-30. 
[34] Han X, Pan J, Buchanan F, Weir N, Farrar D. Analysis of degradation data of poly(1lactide-co-1,d-lactide) and poly(l-lactide) obtained at elevated and physiological temperatures using mathematical models. Acta Biomaterialia 2010;6:3882-9.

[35] Gleadall A, Pan J, Atkinson H. A simplified theory of crystallisation induced by polymer chain scissions for biodegradable polyesters. Polymer Degradation and Stability 2012;97:161620.

[36] Shih C. Chain-end scission in acid catalyzed hydrolysis of poly (D,L-lactide) in solution. Journal of Controlled Release 1995;34:9-15.

[37] Siparsky GL, Voorhees KJ, Miao F. Hydrolysis of polylactic acid (PLA) and polycaprolactone (PCL) in aqueous acetonitrile solutions: Autocatalysis. Journal of Environmental Polymer Degradation 1998;6:31-41.

[38] Buchanan FJ. Degradation rate of bioresorbable materials: Prediction and evaluation. Cambridge, England/Boca Raton [FL]: Woodhead Publishing/CRC Press; 2008.

[39] Gleadall A, Pan J, Kruft M-A. Degradation mechanisms of bioresorbable polyesters, part 2: effects of initial molecular weight and residual monomer. Acta Biomaterialia.

[40] Wiggins JS, Hassan MK, Mauritz KA, Storey RF. Hydrolytic degradation of poly(d,1lactide) as a function of end group: Carboxylic acid vs. hydroxyl. Polymer 2006;47:1960-9. [41] Flory PJ. Principles of Polymer Chemistry. Ithaca, NY: Cornell University Press; 1955. [42] Avrami M. Kinetics of phase change. I General theory. The Journal of Chemical Physics 1939;7:1103-12.

[43] Avrami M. Kinetics of phase change. II Transformation-time relations for random distribution of nuclei. The Journal of Chemical Physics 1940;8:212-24.

[44] Avrami M. Granulation, phase change, and microstructure kinetics of phase change. III. The Journal of Chemical Physics 1941;9:177-84.

[45] Zong X-H, Wang Z-G, Hsiao BS, Chu B, Zhou JJ, Jamiolkowski DD, et al. Structure and morphology changes in absorbable poly(glycolide) and poly(glycolide-co-lactide) during in vitro degradation. Macromolecules 1999;32:8107-14.

[46] Yoon J-S, Jung H-W, Kim M-N, Park E-S. Diffusion coefficient and equilibrium solubility of water molecules in biodegradable polymers. Journal of Applied Polymer Science 2000;77:1716-22.

[47] Li SM, Garreau H, Vert M. Structure-property relationships in the case of the degradation of massive aliphatic poly-( $\alpha$-hydroxy acids) in aqueous media. Journal of Materials Science: Materials in Medicine 1990;1:123-30. 
[48] Li SM, Garreau H, Vert M. Structure-property relationships in the case of the degradation of massive poly( $\alpha$-hydroxy acids) in aqueous media. Journal of Materials Science: Materials in Medicine 1990;1:131-9.

[49] Vey E, Roger C, Meehan L, Booth J, Claybourn M, Miller AF, et al. Degradation mechanism of poly(lactic-co-glycolic) acid block copolymer cast films in phosphate buffer solution. Polymer Degradation and Stability 2008;93:1869-76.

[50] Schliecker G, Schmidt C, Fuchs S, Kissel T. Characterization of a homologous series of d,l-lactic acid oligomers; a mechanistic study on the degradation kinetics in vitro. Biomaterials 2003;24:3835-44. 